\title{
Intravenous immunoglobulins
}

\author{
Of benefit in primary hypogammaglobulinaemia
}

Intravenous immunoglobulin has been used in the United Kingdom for about 10 years, initially as prophylaxis against infections in patients with primary hypogammaglobulinaemia. The rationale was straightforward: a broad range of IgG antibodies from many donors provided protective opsonic, complement fixing, and neutralising antibodies against microbial infection. ${ }^{1}$ Intramuscular gammaglobulin had previously been available to treat patients with primary hypogammaglobulinaemia, but the maximum tolerated dose in adults is about $2 \mathrm{~g}$ a week, whereas the standard dose of intravenous immunoglobulin is about $15 \mathrm{~g}$ every fortnight. Although patients with hypogammaglobulinaemia differ in their requirements for immunoglobulin, the current trend is towards giving higher doses more frequently (that is, weekly) in those who have persistent respiratory infections. Patients with secondary hypogammaglobulinaemia may also benefit from treatment with intravenous immunoglobulin, particularly those with chronic lymphatic leukaemia. ${ }^{2}$ Small studies show that intravenous immunoglobulin reduces the number of infections in children infected with HIV, in premature low birthweight infants, and after bone marrow transplantation, but the results of larger controlled trials are awaited. ${ }^{3}$

Hypogammaglobulinaemia is relatively rare, and most commercial companies making intravenous immunoglobulin have had an eye on potentially larger markets. Their enthusiasm was fired by the serendipitous observation by Paul Imbach, a Swiss paediatrician, that intravenous immunoglobulin increased the platelet count in two hypogammaglobulinaemic children with idiopathic thrombocytopenic purpura. ${ }^{+}$This stimulated much basic work on the interaction of intravenous immunoglobulins with $\mathrm{Fc}$ receptors on phagocytes and on the effect of anti-idiotypic antibodies on the immune network. Intravenous immunoglobulins are now known to block Fc receptors, which may play an important part in preventing the destruction of platelets.

Intravenous immunoglobulins contain anti-idiotypic antibodies directed against a wide range of autoantibodies, although whether these anti-idiotypes switch off the production of autoantibodies in the recipient is uncertain. Whatever the case, intravenous immunoglobulin is now an accepted treatment for idiopathic thrombocytopenic purpura. It also down regulates the production of autoantibodies in those patients with haemophilia who are unfortunate enough to develop autoantibodies against factor VIII. ${ }^{5}$ Small groups of patients with other autoimmune disorders have received intravenous immunoglobulin with promising results. They include patients with myasthenia gravis, pure red cell aplasia, chronic inflammatory demyelinating polyneuropathy, and ophthalmopathy associated with thyroid disease. Patients with rheumatoid arthritis have not benefited. Encouraging results have been reported in other conditions, which may not have an autoimmune basis, such as spontaneous recurrent abortion, intractable childhood epilepsy, myalgic encephalomyelitis, and chronic inflammatory bowel disease. ${ }^{5}$ Controlled studies in the Kawasaki syndrome have convinced most paediatricians that intravenous immunoglobulin should be given early after presentation to reduce the risk of vasculitic complications. The mechanism is unclear but may include down regulation of the production of interleukin 1 by macrophages. ${ }^{67}$

Perhaps the most exciting recent development is the finding that intravenous immunoglobulin may benefit children with severe asthma who are dependent on steroids. ${ }^{4} \mathrm{~A}$ trivial explanation is that intravenous immunoglobulin merely reduces the frequency of respiratory viral or bacterial infections that trigger asthma, but the fall in serum IgE concentrations that accompanies clinical improvement suggests a role for intravenous immunoglobulin in regulating IgE responses.

Cautious clinicians might want evidence that intravenous immunoglobulin is free from contaminating viruses: the material comes from a large pool of plasma collected from up to 2000 donors, with no guarantee that all of them are free from subclinical viral infection, particularly hepatitis $C$. Two major and two minor outbreaks of iatrogenic hepatitis (presumably hepatitis C) have occurred in patients with hypogammaglobulinaemia receiving intravenous immunoglobulin in the past 10 years. ${ }^{8} \mathrm{~A}$ more recent report of hepatitis in a small cluster of patients in Italy receiving an intravenous immunoglobulin preparation with an established safety record suggests that very little margin for safety exists in some of the current manufacturing techniques. The major outbreaks have been associated with a relatively rapid deterioration in liver function and cirrhosis within seven years, suggesting that hepatitis $\mathrm{C}$ follows an unusually aggressive course in patients with hypogammaglobulinaemia. There has been no problem with contamination with hepatitis $B$ virus, presumably because all donors have been adequately screened since intravenous immunoglobulin was introduced. A few patients probably acquired HIV from contaminated intravenous immunoglobulin at the beginning of the AIDS epidemic, before donors could be screened. Fortunately, however, HIV is particularly susceptible to alcohol, and the 
manufacture of intravenous immunoglobulin entails a series of alcohol precipitation steps. Recently, specific techniques have been designed to inactivate a range of viruses ${ }^{9}-$ for example, intravenous immunoglobulins that have been heated or treated with solvent and detergent are currently on trial with some confidence that these procedures will inactivate all hepatitis viruses.

Most licensed intravenous immunoglobulin preparations are well tolerated, causing few short term side effects, although patients with hypogammaglobulinaemia commonly experience mild or moderate reactions during their first few infusions. Many of these patients carry a considerable microbial load before treatment, so it is not surprising that any sudden interaction of infused antibodies with these microbes might release inflammatory mediators, causing fever, rigors, abdominal pain, and sometimes bronchoconstriction and hypotension. As a general rule, infusing intravenous immunoglobulin during acute infection is not advised-it is better to wait until the patient improves with antibiotic treatment. A few patients with hypogammaglobulinaemia have repeated reactions, presumably due to minor contaminants in intravenous immunoglobulin, and changing to a different preparation may be helpful. The overall message, however, is that currently available intravenous immunoglobulins are well tolerated, to such an extent that an increasing number of patients with hypogammaglobulinaemia currently give themselves infusions at home. Guidelines agreed with the Department of Health state that such patients should be trained for home treatment in designated centres, of which there are now seven in the United Kingdom.

Life threatening anaphylactic reactions may occur in one important but rare combination of events. Patients with selective deficiency of IgA may develop anti-IgA antibodies in their serum after their first exposure to blood or blood products containing IgA. Routine screening is necessary to identify these patients, who should then be given preparations of intravenous immunoglobulin containing minimal amounts of IgA. A safe policy is to ensure that all patients receiving intravenous immunoglobulin are supervised by experienced staff for at least the first four infusions.

Aggressive marketing is putting pressure on pharmacies to stock only one commercial preparation of immunoglobulin, and some patients with hypogammaglobulinaemia have already been switched from one type to another, sometimes without consultation with their doctors. Any tendency to regard intravenous immunoglobulin as a generic preparation should be discouraged as manufacturing procedures vary and the final product is only crudely standardised. Further outbreaks of hepatitis, which could not be linked to any particular preparation, could leave patients at a considerable disadvantage if they wished to claim for compensation. Doctors should acquaint themselves with the broad principles of the manufacturing process so that they know whether they are using material that has been subjected to viral inactivation procedures (so called third generation intravenous immunoglobulins) or second generation products, which from experience are considered to be safe. For those who might participate in clinical trials of new preparations, it is wise to dig a little deeper into the background and infrastructure of the manufacturer-and to feel uneasy if the company concerned has not had previous experience in making a safe intravenous immunoglobulin.

Exciting developments in treatment with intravenous immunoglobulin should take place in the 1990s. Multicentre trials are needed to test efficacy in some of the rarer autoimmune diseases, and for the commoner disorders like myalgic encephalomyelitis or asthma a double blind trial will probably be necessary. One possible benefit of the new cost cutting NHS is that doctors will be discouraged from using expensive intravenous immunoglobulins empirically until the results of formal trials are available.

A D B WEBSTER

Consultant Physician,

Immunodeficiency Research Group,

Clinical Research Centre,

Harrow,

Middlesex HA1 3UJ

Cunningham-Rundles C. Immunoglobulin replacement therapy. In: Webster ADB, ed. Immunodeficiency and disease. London: Kluwer Academic, 1988:43-60.

Cooperative Group for the Study of Immunoglobulin in Chronic Lymphocytic Leukaemia. Intravenous immunoglobulin for the prevention of infection in chronic lymphocytic leukemia: a Intravenous immunoglobulin for the prevention of infection in chro

3 National Institutes of Health Office of Medical Applications of Research. NIH consensus statement on intravenous immunoglobulin. Bethesda, Maryland: US Department of Health and Human Services, intravenous immunoglobulin. Bethesda, Maryland: US Depart

1990. (NIH Consensus Development Conference 8 (5).)
Imbach P, Barundun S, d'Apuzzo V, Baumgartner C, Hirt A, Morell A, et al. High-dose intravenous

4 Imbach P, Barundun S, d'Apuzzo V, Baumgartner C, Hirt A, Morell A, et al. High-dose intravenous gammaglobulin for idiopathic thrombocytopenic purpura in childhood. Lancet 1981;1:1228-31.

5 Imbach P, ed. Immunotherapy with intravenous immunoglobulin. London: Academic Press, 1990.

6 Leung DYM, Cotran RS, Kurt-Jones E, Burns JC, Newberger JW, Pober JS. Endothelial cell activation and high interleukin-1 secretion in the pathogenesis of acute Kawasaki disease. Lancel
1989;ii: 1298-301.

Neuberger JW, Takahashi M, Beiser AS, Burns JC, Bastian J, Kyung Ja Chung, et al. A sing Intravenous infusion of gammaglobulin as compared with four infusions in the treatment of acute
Kawasaki syndrome. N Engl F Med 1991;324:1633-9. hepatitis by $\mathrm{pH} 4$-treated intravenous immunoglobulin. Vox Sang 1989;57:15-8.

Krijnen HW, Strengers PFW, van Aken W'G, eds. Immunoglobulins. Proceedings of an international symposium. Amsterdam: Central Laboratory of The Netherlands Blood Transfusion Service, 1988.

\section{Nursing: an intellectual activity}

\section{Nurses need heads as well as hands}

There are two ways of looking at nursing: which one you choose determines your whole perspective on the nursing world.

The first sees nursing as a collection of tasks or procedures which require some skill but are initiated and directed by others-particularly doctors - whose function they exist to assist. Because they require skill some training is necessary, but competent performance does not require an understanding of why the task is necessary, how it works, or what its effects will be. Within this view nursing has no knowledge base of its own, nor does it need one: its skills are essentially manual and technical and reflect the knowledge of other disciplines.

The second, quite different, view sees nursing as a particular kind of interpersonal interaction, which has specific goals and involves particular kinds of activities. Its focus is the physical and emotional responses of people to illness, treatment, and disability. Its goal is to enable people (individuals, families, and communities) to maximise their potential for health; to enhance their ability to cope with illness and disability; and to promote physical and mental comfort, healing, and recovery. What distinguishes this perspective from the first is its intellectual component: the use of clinical judgment based on knowledge.

Assessment, planning, implementation, and evaluation all depend on clinical judgment. Assessment necessitates seeking relevant information (by observing, examining, and history 